\title{
Crostacei Decapodi raccolti nella Somalia
}

\author{
DAI DOTTORI STEFANINI E PAOLI
}

E vietata la riproduzione.

La presente nota si riferisce a poche specie di Crostacei De. capodi raccolte dai dottori Stefanini e Paoli durante il loro viaggio nelia Somalia italiana (1913). Benchè nessuna di esse sia nuova, il loro esame non è riuscito privo di interesse. Una femmina di $P e$ naeus carinatus Dana si è prestata ad una discussione sinoni. mica, per la quale mi è stata molto utile la rara opera di $\mathrm{Paul}$ son: "Ricerche sui Crostacei del Mar Rosso "(in lingua russa) pub. blicata a Kiew nel 1875 in numero di 100 esemplari, di cui solo una sessantina esistono ancora ed uno nella Biblioteca di questo Istituto.

Oltre alle specie che descrivo fanno parte della collezione due campioni di Alpheus raccolti sulla spiaggia di Mogadiscio; ma il loro stadio ancora giovanile e la cattiva conservazione mi impediscono di determinarli specificamente.

\section{Penaeus carinatus DANA.}

1852. - Penaeus carinatus Dana (U. S. Explor., Exped. Crustacea).

1868. - P. tahitensis Heller (Reise Osterreich. Freg. Novara, Crustaceen I, Wien).

1875. P. semisulcatus Paulson (Ricerche sui Crostacei del Mar Rosso; Kiew (in lingua russa).

1888. P. monodon (partim). SPence-Bate (Challenger Report, XXVI, Macrura). 
1888. P. semisulcatus (partim) DE MAN (Journ. Linn. Soc. London, XXII).

1892. P. semisulcatus (partim) DE MAN (Max Weber's Zool. Ergebn.).

1893. P. monodon Henderson (Contrib. Indian Carcinology).

1898. P. monodon de Man (Zool. Jahrb. X System).

1900. P. monodon Kishinouye (Journ. Fisheries Bureau, VIII, Tokyo).

1903. P. monodon Nobili (Boll. Mus. Zool. Torino, ni. 452 e $455)$.

1906. P. semisulcatus AlcoçK (Catal. Indian Decap. Crustacea, III, Macrura).

1911. P. carinatus DE MAN (Siboga-Expeditie, XXXIX a, Peneidae).

1915. P. carinatus Kemp (Mem. Ind. Mus, V).

Un solo esemplare femina, catturato il 21 luglio 1913 presso la vecchia foce del Giuba, lungo circa 14 centimetri.

Lo scudo dorsale è lungo $\mathrm{mm}$. 54, la carena dorsale comincia fin dal margine posteriore; il rostro sporge di mm. 25; il primo dente si trova a $36 \mathrm{~mm}$. dall'estremità del rostro. I denti sono in numero di 6 superiormente, e solo 2 inferiormente, entrambi situati fra l'estremità del rostro e l'ultimo dente superiore.

L'estremita del rostro supera appena il livello a cui giungono le squame antennali e notevolmente l'estremità dei peduncoli antennulari, i quali sono lunghi mm. 16 məntre il loro flagello superiore è lungo $29 \mathrm{~mm}$. e l'inferiore $28 \mathrm{~mm}$.

L'ultimo pajo di pereopodi non possiede traccia di esopodite, mentre tutte le altre paja hanno un esopodite bene sviluppato.

Il pleon col telson è lungo $\mathrm{mm}$. 84. La carena del pleon co. mincia a metà del $4^{\circ}$ segmento e continua accentuandosi nel $5^{\prime \prime}$ e e nel $6^{\circ}$. Il margine laterale posteriore del $1^{\circ}$ e del $2^{\circ}$ segmento pleonale possiede una piccola ma netta rientranza, la quale manca nel $3^{\circ}$ segmento e si ritrova accentuala nel $4^{\circ}$ e nel $5^{\circ}$.

Il thelycum è costituito da due laminette appartenenti all' ul. timo sternite toracico i cui margini posteriori rimangono nascosti dal margine anteriore dello sternite stesso, mentre la loro estremità anteriore rimane libera e arrotondata, e i margini laterali interni rimangono strettamente accostati nella metà posteriore e si allontanano anteriormente: dall' intervallo che cosi si forma sporge un processo tagliente che nasce dal centro di una placchetta qua- 
drangolare arrotondata, più lunga che larga, posta sulla linea mediana del penultimo sternite.

Pencueus carinatus è abbastanza diffuso sulle coste asiatiche dell' Indo-Pacifico. È piuttosto raro nella baja di Tokyo e nella baja di Ise nel Giappone (Kishrnoure); si trova a Makassal e ad Atjeh (De Man), a Singapore (Dana, Nobili) a Mergui (De Man, Alcock); è comune in tutte le coste dell' India e a Ceylon. Kemp l'ha riscon. trato anche nel lago Chilka. A queste località è ancora da aggiungere la costa della Somalia, e il Mar Rosso (Paulson). Tale di stribuzione come si vede è identica a quella di $P$. semisulcatus DeHAan, specie strettamente affine con la quale $P$. carinatus è stiato lungamente confuso.

La sinonimia di questa specie infatti è molto intricata. Nel 1849 DehaAn descrisse un Penaeus giapponese che chiamò $P$. semisulcatus. Ma i caratteri dati dall'autore sono molto generali tanto che potevano valere ugualmente per una specie molto affine che tre anni dopo DANA descriveva col nome di $P$. carinatus. A cagione delle descrizioni date da questi due autori e dello sforzo fatto da aitri di identificare il Penaeus monodon di FABRICIUs con l'una 0 l'altra di tali forme, sono state vicendevolmente indicate con lo stesso nome specie diverse e con vari nomi la stessa specie, finchè DE MAN nel 1911 pensò di togliere dalla sinonimia come irriconoscibile $P$. monodon FABricius e, prendendo nuovaniente in esame il tipo di $P$. semisulcatus DehaAn conservato nel Museo di Leida, stabilì fermamente che $P$. semisulcatus DEHA tN è diverso da $P$. carinatus DANA; e la differenza sta in ciò, che mentre in $P$. semisulcatus DeHAAN I'ultimo pajo di zampe toraciche è sempre provvisto di un esopodite bene sviluppato, tale esopodite manca invece in $P$. carinatus DANA.

Nel 1868 Heller indicò coi nomi di P. semisulcatus, P. mono. don e $P$. carinatus vari campioni raccolti dalla Nave "Novara "; ma non essendo le determinazioni corredate da alcuna aggiunta descrittiva nè da figura, è dubbio qual posto debbano esse tenere nella sinonimia. Contemporaneamente Helder descriveva $P$. tahitensis; ma come con ragione fece pui notare Spence-Bate la descrizione HeLler non corrisponde affatto alla figura. Egli infatti dice " crista dorsali 8-9 dentata, margine inferiore edentulo " mentre nella figura si vedono rappresentati tre denti al margine inferiore del rostro; e dice ancora " flagella pedunculis longiora et ultimum rostri dentem attingentia "mentre nella figura appaiono di dimen- 
sioni alquanto maggiori. Basandosi sopratutto sulla figura SPenceBate riportò $P$. tahitensis HeLLer a $P$. carinatus Dana.

Nel 1875 Paulson, nella prima parte della sua interessante monografia sui Crostacei del Mar Rosso, indica col nome di $P$. semisulcatus, riferendolo alle specie di DeHAan e di Helcer un individuo femina di cui descrive e rappresenta in figura il thelycum. Così egli dice: " fia il quarto paio di zampe si trova nella femina una prominenza quadrata con una sporgenza a braccio, la quale entra nell' intervallo formato da due piastre del quinto paio di zampe „. Ora DeHAAN descrive il thelycum semplicemente con queste parole: "Sternum feminarum inter pedes quintos rima media continua divisum et reflexum „. Non mi pare dunque che l'identificazione di Paulson sia stata esatta, molto più che la descrizione e la figura da esso date concordano perfettamente con quelle del mio esemplare di $P$. carinatum, mentre la descrizione di DEHAAN ben si adatta a $P$. semisulcatus. $P$. semisulcatus PAUrson deve dunque passare in sinonimia di $P$. carinatus DANA e non di $P$. semisulcatus DEHAAN.

Spence-Bate nel 1888 diede la seguente sinonimia: Penaeus monodon Fabricius $=P$. monodon Milne-Edwards $=P$. semisulcatus De HAAN $=P$. carinatus DANA $=P$. monodon SPENCE-BAte. Dall'esame delle figure però mi accorgo che egli possedeva individui di due specie diverse. Nella fig. 1 della Tavola XXXIV è rappresentato un maschio che possiede l'esopodite dell'ultimo pajo di zampe bene sviluppato e che perciò deve ascriversi alla specie $P$. semisulcatus, mentre nella fig. 1" e nella 1", della stessa tavola sono rispettivamente rappresentati un maschio ed una femina privi dell'esopodite del $6^{\circ}$ paio di pereopodi e che sarebbero senz'altro da identificarsi con $P$. carinatum DANA, se il thelycum della femina non presentasse i caratteri dati per $P$. semisulcatus. Io però credo fermamente che la fig. 1" rappresenti un maschio di P. carinatum e la fig. 1" "una femina di P. semisulcatus con omissione, per errore o trascuratezza nel disegno, dell'esopodite del $5^{\circ}$ paio di pereopodi. È evidente che SPENCE-BAte non teneva in conto il carattere di presenza o mancanza di tale esopodite, l'unico che serve veramente per la distinzione di $P$. semisulcatus da $P$. carinatus. Egli inoltre non solo considera come sinonimi $P$. semisulcatus DEHAAN e $P$. carinatus Dana, ma li riferisce a $P$. monodon, attribuendo a questo nome il diritto di priorità. $P$. monodon FABRICIUS però è da cancellarsi perchè inidentificabile e $P$. monodon MILne-Edwards è basato sull'esame di individui troppo giovani. $P$. monodon Spence-Bate 
deve dunque passare in parte in sinonimia di $P$. semisulcatus $e$ in parte di $P$. carinatus.

Alcock ammette le due specie $P$. monodon e $P$. semisulcatus, passando $P$. carinatus in sinonimia di $P$. monodon Spence-Bate. Egli però parte da basi fondamentalmente erronee, attribuendo a $P$. semisulcatus la mancinza dell'esopodite del $5^{0}$ paio di pereopodi e a P. monodon la presenza di esso. La figura (Tav. I, fig. 1 b) che egli dà del thelycum di $P$. monodon (FABRICIUS) ALcock corrisponde perfettamente a quella di $P$. monodon (Fabricius) Spence-Bate e alla descrizione fatta da DeHaAn per $F$. semisulcatus.

Spetta a De Man l'aver dimostrato che la specie descritta da Alcock come $P$. semisulcatus DehaAn è invece di $P$. carinatus DANa, che la specie descritta dallo stesso autore col nome di $P$. monodon FABRicius è molto probabilmente identica a $P$. semisulcatus Dehana, che la specie descritta da Kishinoure col nome di $P$. ashiaka è identica a $P$. semisulcatus Dehan.

De Man stabilisce le seguenti sinonimie:

Penaeus semisulcatus DE HaAN.

1849. P. semisulcatus De HaAN.

1880. P. semisulcatus De Man.

1890. P. semisulcatus Ortmanit.

1888. P. monodon Spence-Bate.

? 1905. P. monodon Stebbing.

? 1906. P. monodon Alcock.

1900. P. ashiaka Kishinouye.

1902. P. ashiaka R ITHBun.

1903. P. ashiaka Nobili.

Penaens carinatus DANA.

1852. P. carinatus DANA.

1888. P. semisulcatus De MAN.

1892. P. semisulcatus De MAN.

1893. P. monodon Henderson.

1898. P. monodon DE MAN.

1900. P. monodon Kishrnouye.

1903. P. monodon NobiLt.

1906. P. semisulcatus A Acock.

Secondo quanto ho detto in precedenza, io credo che $P$. mono. don Spence Bate debba passarsi in sinonimia tanto di $P$. semisulcatus De HaAN che di P. carinatus Dana, che P. monodon Alcock sia senz'altro uguale a $P$. semisulcatus DE HAAN, che $P$. semisulcatus 
Paulson sia lo stesso che $P$. carinatus Dana e che $P$. tahitensis Helder corrisponda pure a $P$. carinatus Dana.

\section{Palaemon dolichodactylus HILGENDORF}

1878. P. dolichodactylus Hilgendorf (Monatsber. Königl. Akad. Wissensch. Berlin).

1900. P. dolichodactylus Coutik̀ne (Annn. Sc. Nat. Zool. (8) XII.

1913. P. (Parapalaemon) dolichodactylus Catiman (Proc. Zool. Soc. London; 1913).

Quattro maschi lunghi mm. 55.64.

Lo scudo dorsale è finemente, ma chiaramente cosparso di fitte sporgenze papillari, ed è lungo $24.28 \mathrm{~mm}$. Il rostro sporge di 10-11 mm., possiede 15-17 denti superiormente, 3 denti inferiormente. La carena dorsale comincia a metà dello scudo, col primo dente del rostro, Le squamme antennali e il rostro giungono circa allo stesso livello ed entrambi oltrepassano i peduncoli antennulari.

Il pereopodi del $1^{\circ}$ paio sono molto vari per grandezze. Il $1^{\circ}$ pereopodo destro di uno dei miei esemplari è lungo $\mathrm{mm} .79 \mathrm{di}$ cui mm. 40.5 spettano alla chela, la quale nella sola regione palmare è ricoperta di un feltro fittissimo, mentre lo rimanenti regioni hanno superficie aspra per numerose papille ed ispida di setole lunghette specialmente sul margine interno e alle due punte della chela: esso corrisponde perfettamente al disegno di Hilgendorf. Il dito mobile possiede sul margine inferiore con 23 denti allungati, il dito immobile possiede nel margine superiore 14 di tali denti. Il primo pereopodo sinistro di un altro campione è lungo $55 \mathrm{~mm}$. di cui $28 \mathrm{~mm}$. spettano alla chela: il dito mobile possiede 21 denti e l'immobile 14 il feltro della regione palmare è appena accennato. I pereopodi del $1^{0}$ paio quando hanno una lunghezza inferiore ne ho esaminati di mm. 40, 34, 33, 27 - non posseggono il feltro nella regione palmare, ma sono in tutta la loro superficie ispidi di setole vistose.

La specie è diffusa nel littorale africano dell'Oceano Indiano. Gli individui da me esaminati provengono dal Giuba (9 gennaio 1913).

\section{Palaemon mossambicus HILGENDORF.}

1873. C. mossambicus Hilgendorf (Monatsber. Königl. Akad. Wissensch. Berlin).

Lunghezza mm. 94 di cui $\mathrm{mm} .45$ spettano al cefalotorace col 
rostro, il quale sporge di mm. 21 e possiede 12 denti superiormente e 5 denti inferiornente: gli ultimi due denti superiori si trovane più innanzi dell'ultimo inferiore, il primo dente superiore si trova a $25 \mathrm{~mm}$. dall'apice del rostro. Il rostro supera di qualche millimetro le squamme antennali, e queste l'estremità di peduncoli antennulari. Dei pereopodi dal primo paio, il sinistro è lungo $120 \mathrm{~mm}$, di cui 45 spettano alla chela, il destro mm. 65 , di cui 24 spettano alla chela: entrambi sono uniformemente ricoperti da una lievissima peluria. Il dito immobile della chela maggior possiede un dente conico grossetto presso la base, ed una doppia serie longitudinale di minuti denti immersi nella pelurie, che solo in vicinanza di essi è più cospicua, e termina con una spina volta all'insù; il dito mobile del mio esemplare è in rigenerazione. La chela minore presente; sia nel dito mobile che nel dito immobile, dei denticoli assai minuti e poco regolari: l'estremità dei diti è come a becco.

Un solo maschio proveniente dalla vecchia foce del Giuba. La specie si ritrova in tutto il littorale africano dell'Oceano Indiano.

\section{Potamon (Potamonautes) dubius (CAPELLO).}

1873. Thelphusa dubia CAPELLo (Jorn. Sc. Lisboa, IV). 1887. 1887. (7), IV). Ozorio (Jorn. Sc. Lisboa, XI).

1897. Potamon (Potamonautes) dubium Ortmann (Zool. Jahrb., Syst, X).

1900. Mus., XXII).

dubius Rathbun (Proc. U. S. Nat.

Ascrivo alla specie Potamon (Potamonautes) dubius una femina catturata presso la vecchia foce del Giuba.

Scudo dorsale lungo mm. 22,5, largo mm. 29,5. Fronte abbastanza larga; margine frontale integro, con leggera insenatura mediana; si passa con curva molto regolare dal margine anteriore libero al margine sopraccigliare. Margini orbitali finemente crenulati; spina extraorbitale acuta con margine interno crenato e margine esterno leggermente dentato. Una spina epibranchiale acuta con margini leggermente dentati; la dentellatura si continua esternamente per un po' sul margine laterale dello scudo per svolg irsi dorsalmente e all'interno fra la regione branchiale anteriore e la posteriore. La cresta postfrontale è molto netta e si estende senza interruzione dal solco mesogastrico fino alla base dei denti epibranchiali. La re- 
gione mesogastrica dello scudo è separata dalle regioni epigastriche e dalle regioni branchiali mediante solchi poco profondi, ma abbastanza distinti, mentre manca ogni solco di separazione fra la regione mesogastrica e le regioni protogastriche. La regione urogastrica è lunga circa la metà della sua larghezza ed è completamente separata dalle regioni limitrofe mediante solchi poco profondi. Così pure la regione cardiaca, un po' più larga che lunga.

I chelipedi suno piuttosto corti e gracili rispetto agli altri pereopodi; il chelipede sinistro è maggiore del destro.

Chelipede sinistro, lunghezza. . . . . mm. 30

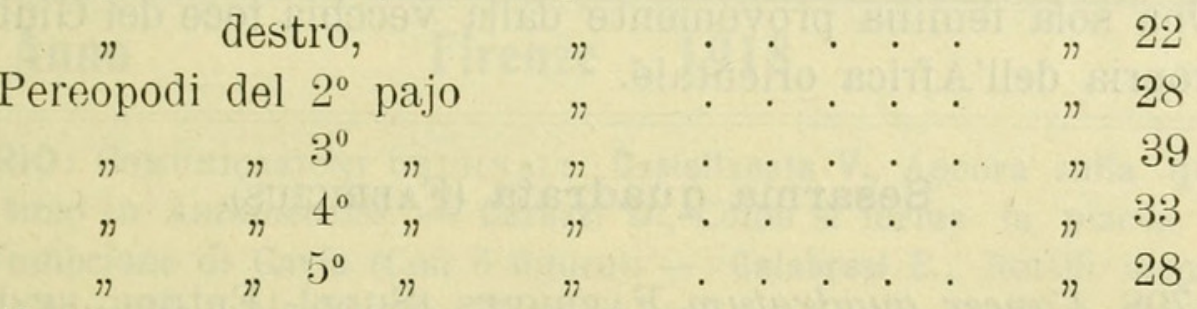

Dalla descrizione non appare dubbio the la specie, sia riferibile a Potamonautes dubius (CAPELLo); le deviazioni dal tipo rientrano benissimo nei limiti delle variazioni individuali 0 anche delle variazioni geografiche. Anche Potamonautes dubius Jallae (NoBILI) (Bull. Mus. Zool. Anat. Comp. Torino, XI, 1896) non è che una variazione geografica della specie descritta da CAPELlo. È notevole, che mentre P. dubius CAPELlo e P. dubius Jallae Nobili erano stati finora descritti per l'Africa occidentale, la specie rimane anche accertata per la Somalia.

Ci)me proprio della Somalia è indicato P. Bottegoi de Man (Ann. Mus. Civ. Storia Nat. Genova, (2) XIX (XXXIX, 1898) che differisce principalmente da $P$. dubius per avere la cresta postfrontale che non si riunisce al dente epibranchiale, ma che si arresta prima di giungervi e torna per breve tratto all'indietro. Inoltre, mentre il margine frontale anteriore di $P$. Bottegoi è convesso, quello di $P$. dubius presenta invece una lieve insenatura.

\section{Deckenia imitatrix HILGENDORF.}

1869. Deckenia imitatrix Hilgendorf (Gesell. naturf. Fr. Berlin, 1868).

1869. D. imitatrix Hulgendorf (Baron C. Claus von der Decken's Reisen in Ost. Africa, III, 1, Crustaceen).

1869. D. imitatrix. Milne-Edwards (Nouv. Arch. Mus. Hist. Nat., V).

1887. D. imitatrix Milne-Edwards (Ann. Sc. Nat., Zool. (7), IV). 
1893. D. imitatrix Milne-Edwards e Bouvier (Ann. Sc. Nat,, Zool., (7) XV.

1897. D. imitatrix Ortmann (Zool. Jahrb., Syst., X).

1898. " " Hilgendorf (Die Thierwelt Deutsch Ost. Afr., IV).

1898. D. imitatrix De Man (Ann. Mus. Civ. Stor. Nat. Genova (2) XIX).

1903. D. imitatrix Ortmann (Proc. Amer. Philos. Soc., XLI).

1906. D. imitatrix Rathbum (Nouv. Arch. Mus. Hist. Nat. (5) VIII.

Una sola femina proveniente dalla vecchia foce del Giuba. Specie propria dell'Africa orientale.

\section{Sesarma quadrata (FABRICIUS).}

1798. Cancer quadratum Fabricius (Suppl. Entom. syst.)

1853. Sesarma quadrata Milne-EdwaRds (Hist. nat. des crust. XI) (rust).

1849. Grapsus (Pachysoma) pictus DE HAAN (Fauna Japonica,

1849. Grapsus (Pachysoma) affinis DE HAAN (Fauna Japonica, Crust).

1843. Sesarma picta Krauss (Südafrikanischen Crustaceen, Stuttgart).

1869. Sesarma quadrata Hilgendorf (Bar. Carl Claus von del Decken's Reisen in Ost. Afrika; III, 1, Crustaceen).

1869. Sesarma quadratum Milne-Edwards (Nouv. Arch. du Museum, V).

1802. Ocypode plicata Bosc (Hist. des Crustacés, I).

Due campioni maschi provenienti dalla foce del Giuba (gennaio 1913).

Firenze, 29 luglio 1918.

Istituto di Zoologia degli Invertebrati.

Cosimo Cherubini, Amministratore-responsabile.

Firenze, 1918. - Tip. L. Niecolai, Via Faenza, 52. 


\section{$2 \mathrm{BHL}$ Biodiversity Heritage Library}

Paoli, Stefanni E. 1918. "Crostacei Decapodi raccolti nella Somalia." Monitore zoologico italiano 29, 100-108. https://doi.org/10.5962/bhl.part.1197.

View This Item Online: https://www.biodiversitylibrary.org/item/37008

DOI: https://doi.org/10.5962/bhl.part.1197

Permalink: https://www.biodiversitylibrary.org/partpdf/1197

\section{Holding Institution}

MBLWHOI Library

Sponsored by

MBLWHOI Library

\section{Copyright \& Reuse}

Copyright Status: NOT_IN_COPYRIGHT

This document was created from content at the Biodiversity Heritage Library, the world's largest open access digital library for biodiversity literature and archives. Visit BHL at https://www.biodiversitylibrary.org. 